\title{
Dynamical Properties of a Herbivore-Plankton Impulsive Semidynamic System with Eating Behavior
}

\author{
Yufei Wang, ${ }^{1}$ Huidong Cheng $\mathbb{D}^{1}{ }^{1}$ and Qingjian $\mathbf{L i}^{2}$ \\ ${ }^{1}$ College of Mathematics and System Sciences, Shandong University of Science and Technology, Qingdao 266590, \\ Shandong, China \\ ${ }^{2}$ College of International Office, Shandong University of Science and Technology, Qingdao 266590, Shandong, China
}

Correspondence should be addressed to Huidong Cheng; chd900517@sdust.edu.cn

Received 29 December 2019; Accepted 9 March 2020; Published 28 April 2020

Academic Editor: Oveis Abedinia

Copyright (c) 2020 Yufei Wang et al. This is an open access article distributed under the Creative Commons Attribution License, which permits unrestricted use, distribution, and reproduction in any medium, provided the original work is properly cited.

In this paper, an impulsive semidynamic system of the relationship between plankton and herbivore is established, and the Poincare map method is used to extend the new properties of the model. We define the Poincare map of the impulsive point series in phase concentration and analyze the characteristics. A comprehensive and detailed analysis of the periodic solution is performed. In addition, the numerical simulations illustrate the correctness of our arguments. The results show that plankton and herbivore can survive stably under effective control.

\section{Introduction}

In terrestrial and aquatic ecosystems, the energy conversion and nutrient cycle between plankton and herbivores play an important role [1]. Since plankton can provide energy for herbivores, studies on the population model of plankton and herbivores have drawn increasing attention from scholars [2-4]. In Zhong et al.'s paper [5], the interaction model of nutrient solution, plankton, and herbivores was established, and the limit cycle and other dynamic properties were studied. Sharma et al. [6] put forward a predator model of plankton and herbivores, made a qualitative analysis of the model system, discussed the stability of the equilibrium point, and explored ways to maintain the ecological balance of the population at different harvest levels.

Recently, the impulsive semidynamical system with threshold has been widely applied [7-11], such as in the diagnosis and treatment of the antivirus system [12-15], the integrated control strategy of ecological resources and plant diseases and insect pests [16-20], vaccination strategies, and epidemic control [21-25]. The research and application of geometric theory in pulsed semidynamic systems have also yielded good results [26-30]; the existence, uniqueness, and stability of system periodic solutions are discussed. Also, the successor function, Lyapunov function, and coincidence theory were used to study the dynamics of biological systems [31-36]. However, with the further development of the state feedback control model and the complexity of global dynamics, there are still many unresolved problems in model research. For example, when the control parameters are changed, the global dynamics are not well processed; the periodic solutions of the model are not thoroughly studied; and the biological meanings of the complex dynamics are not well analyzed and revealed. In order to solve these problems, more advanced qualitative techniques and new methods are needed to reveal the complete dynamic properties and control strategies of biological dynamic systems. Therefore, we propose a herbivore-plankton pulse semidynamic system, which uses the Poincaré map method to comprehensively analyze and study the complex dynamics of the model.

In previous studies, the main assumptions are the number of organisms fished or destroyed during the control process is a linear relationship with the population density or number; when the biological populations reach the threshold, the number of natural enemies released during the pulse is constant and regardless of density. However, in actual situations, in order to better control the biological 
population and obtain benefits, it is necessary to make appropriate control in combination with factors such as biological population density and fishing rate. Therefore, in this paper, for the proposed herbivore-plankton impulsive semidynamic system, the amount of plankton released is a function of its density, and the number of herbivores caught is related to the maximum fishing rate. This allows both plankton and herbivores to be effectively controlled, more in line with actual conditions.

This article is arranged in the following sections: in Section 2, the useful definition of the pulsed semidynamic system and related lemmas are presented. In Section 3, we introduce the plankton-herbivore model and give its qualitative properties. The definition of Poincaré map and its main properties are studied in Section 4. In Section 5, the stability conditions of the boundary periodic solution and the order-1 periodic solution are given, and the existence of the order- $k$ $(k \geq 2)$ periodic solution is further proved. Finally, the numerical simulation is performed to verify the conclusion.

\section{Preliminaries}

In practical biological problems, state feedback control strategies can be broadly defined, usually modeled by impulsive semidynamic systems. Control strategies (harvesting, applying pesticides, treatment, etc.) are only implemented when a particular species attains the previously defined threshold. In many papers, a common assumption is that all solutions from a phase set must undergo an unlimited number of pulses. In order to better research the paper, we propose the following useful definitions and lemma:

Definition 1. A generalized plane pulse semidynamic system with the state-dependent feedback control can be depicted as the following:

$$
\left\{\begin{array}{c}
\frac{\mathrm{d} p(t)}{\mathrm{d} t}=F(p, y), \\
\frac{\mathrm{d} y(t)}{\mathrm{d} t}=G(p, y), \\
\Delta p(t)=\alpha(p, y), \\
\Delta y(t)=\beta(p, y),
\end{array}\right\}(p, y) \in M,
$$

where $F, G, \alpha$, and $\beta$ are continuous funtions and $M \subset R^{2}$ represents the impulsive set. $(p, y) \in R^{2}, \Delta p=p^{+}-p$, and $\Delta y=y^{+}-y$. For any $w(p, y) \in M, w^{+}$indicates the impulsive point; thus, the impulsive function $I$ is defined as $w^{+}=I(\omega)=(p+\alpha(p, y), y+\beta(p, y))=\left(p^{+}, y^{+}\right) \in R^{2}$.

We define $N=I(w)$ as the phase set; then, for all $w \in M, I(w)=w^{+} \in N$, while $N \cap M=\varnothing$.

Define $\left(X, \Pi, R_{+}\right)$or $(X, \Pi)$ as the semidynamical system, then $X$ represents a metric space and $R_{+}$represents all nonnegative reals. When $w \in X$, the function $\Pi_{w}(t)=\Pi(w, t)$ is clearly continuous such that $\Pi(w, 0)=w$ and $\Pi(\Pi(w, t), s)=\Pi(w, t+s)$ for all $w \in X$ and $t, s \in R_{+}$. The set $U^{+}=\left\{\Pi(w, t) \mid t \in R_{+}\right\}$is described as the positive orbit of $w$. For any $t \geq 0$, we have $V(w, t)=\{z: \Pi(z, t)=w\}$, and for all set $M \subset X$, then $M^{+}(w)=U^{+}(w) \cap M-\{w\}$.

Definition 2. A trajectory $\Pi_{w}$ in $(X, \Pi, M, I)$ is called the periodic of period $T_{k}$ and order $k$ if there are integers $m \geq 0$ and $k \geq 1$ which make $k$ the smallest integer of $w_{m}^{+}=w_{m+k}^{+}$ and $T_{k}=\sum_{i=m}^{m+k-1} \phi\left(w_{i}\right)=\sum_{i=m}^{m+k-1} s_{i}$.

More details on the concept and nature of the impulsive semidynamical system can be found in [37-41].

Lemma 1. The order- $k$ periodic solution $(p, y)=(\xi(t), \eta(t))$ of system

$$
\begin{aligned}
\frac{\mathrm{d} p(t)}{\mathrm{d} t} & =F(p, y), \frac{\mathrm{d} y(t)}{\mathrm{d} t}=G(p, y), \\
p^{+} & =p+\alpha(p, y), y^{+}=y+\beta(p, y),
\end{aligned}
$$

is orbitally asymptotically stable and enjoys the property of the asymptotic phase if the Floquet multiplier $\chi_{1}$ satisfies the condition $\left|\chi_{1}\right|<1$, where

$$
\chi_{1}=\prod_{k=1}^{q} \Delta_{k} \exp \left(\int_{0}^{T}\left[\frac{\partial F}{\partial p}(\xi(t), \eta(t))+\frac{\partial G}{\partial y}(\xi(t), \eta(t))\right] \mathrm{d} t\right)
$$

with

$$
\Delta_{k}=\frac{F_{+}((\partial \beta / \partial y)(\partial \Phi / \partial p)-(\partial \beta / \partial p)(\partial \Phi / \partial y)+(\partial \Phi / \partial p))+G_{+}((\partial \alpha / \partial p)(\partial \Phi / \partial y)-(\partial \alpha / \partial y)(\partial \Phi / \partial p)+(\partial \Phi / \partial y))}{F(\partial \Phi / \partial p)+G(\partial \Phi / \partial y)}
$$

where $F, G, \partial \alpha / \partial p, \partial \alpha / \partial y, \partial \beta / \partial p, \partial \beta / \partial y, \partial \Phi / \partial p, \partial \Phi / \partial y$ are calculated at the point $\left(\xi\left(t_{k}\right), \eta\left(t_{k}\right)\right), F_{+}=F\left(\xi\left(t_{k}^{+}\right), \eta\left(t_{k}^{+}\right)\right)$, and $G_{+}=G\left(\xi\left(t_{k}^{+}\right), \eta\left(t_{k}^{+}\right)\right)$. And $\Phi$ is continuously differentiable with respect to $p, y .(p, y) \notin M$ is equivalent to $\phi(p, y) \neq 0$.

\section{Herbivore-Plankton Interaction Model and Its Properties}

Ntr et al. [42] proposed the following herbivore-plankton interaction model: 


$$
\left\{\begin{array}{l}
\frac{\mathrm{d} P(t)}{\mathrm{d} t}=P(t)\left(K-P(t)-\frac{B Y(t)}{C+P(t)}\right), \\
\frac{\mathrm{d} Y(t)}{\mathrm{d} t}=D Y(t)\left(\frac{P(t)}{C+P(t)}-A Y(t)\right),
\end{array}\right.
$$

where $P$ and $Y$ are the density of plankton and herbivores, respectively. $K$ is the constant carrying capacity without herbivores, $D$ is the rate of biological conversion, $B /(C+P)$ indicates the function response of the herbivore to change in the density of plankton, $C$ is the semisaturation constant, and $B$ represents the maximum consumption of herbivores. For more biological definitions, see [42].

We nondimensionalize system (5) and get the following state feedback control model [43]:

$$
\left\{\begin{array}{l}
\frac{\mathrm{d} p(t)}{\mathrm{d} t}=p(t)\left(k-p(t)-\frac{y(t)}{1+p(t)}\right) \\
\frac{\mathrm{d} y(t)}{\mathrm{d} t}=y(t)\left(\frac{p(t)}{1+p(t)}-a y(t)\right), \\
\Delta p(t)=-\alpha p(t), \\
\Delta y(t)=-\beta y(t),
\end{array}\right\} y=\bar{y},
$$

where $p$ and $y$ denote the density of plankton and herbivore at time $t$, respectively, and $a$ indicates the effect of density dependence on herbivores. $\alpha p$ and $\beta y$ indicate the quantity of plankton and herbivore reduced by catching or exhaustion, respectively. System (6) shows that when the quantity of herbivores reaches certain threshold $\bar{y}$, without considering the quantity of plankton, harvesting both herbivores and plankton causes excessive depletion of plankton and leads to a lack of food for herbivores, which in turn affects their production. And at this time, the control of plankton and herbivores is only related to the density, but in actual situations, we need to control the biological population based on the fishing rate and actual factors.

Therefore, we establish the state feedback control model as follows:

$$
\left\{\begin{array}{l}
\frac{\mathrm{d} p(t)}{\mathrm{d} t}=p(t)\left(k-p(t)-\frac{y(t)}{1+p(t)}\right), \\
\frac{\mathrm{d} y(t)}{\mathrm{d} t}=y(t)\left(\frac{p(t)}{1+p(t)}-a y(t)\right), \\
\triangle p(t)=p\left(t^{+}\right)-p(t)=\frac{\tau}{1+\theta p(t)}, \\
\triangle y(t)=y\left(t^{+}\right)-y(t)=-\frac{\delta y^{2}(t)}{y(t)+\beta},
\end{array}\right\} p>\bar{p},
$$

system (7) shows that whenever the quantity of plankton reaches certain threshold $\bar{p}$, people will catch herbivores and increase the amount of plankton to $(1-\delta y(t) / y(t)+\beta) y(t)$ and $p(t)+\tau / 1+\theta p(t)$, respectively, where $\delta$ represents the maximum fishing rate, $\tau$ indicates the maximum amount of plankton added, and $\theta$ represents the morphological parameter. For more definition of biological parameters, see [44]. Define $f(y)=(1-(\delta y / y+\beta)) y$, and by calculating, we get the function $f(y)$ is increasing in $(0,+\infty)$.

The qualitative analysis of system (8) is discussed as follows when system (7) has no pulse:

$$
\left\{\begin{array}{l}
\frac{\mathrm{d} p(t)}{\mathrm{d} t}=p(t)\left(k-p(t)-\frac{y(t)}{1+p(t)}\right), \\
\frac{\mathrm{d} y(t)}{\mathrm{d} t}=y(t)\left(\frac{p(t)}{1+p(t)}-a y(t)\right) .
\end{array}\right.
$$

Theorem 1. The extinction equilibrium point $O(0,0)$ of system (8) is unstable, the boundary equilibrium point $D_{0}(k, 0)$ is the saddle point, and $D^{*}\left(p^{*}, y^{*}\right)$ is the internal equilibrium point [43] (Figure 1).

(i) If $\left(H_{1}\right): k<2 p^{*}+2$, then internal equilibrium point $D^{*}$ is stable

(ii) If $\left(\mathrm{H}_{2}\right): k>2 p^{*}+2$, then internal equilibrium point $D^{*}$ is unstable and has a limit cycle

In addition, Hopf branch occurs in the case of $k=2 p^{*}+2$, where $p^{*}$ meets the following condition:

$$
\begin{aligned}
& p^{*}=a\left(1+p^{*}\right)^{2}\left(k-p^{*}\right), \\
& y^{*}=\frac{p^{*}}{a\left(1+p^{*}\right)} .
\end{aligned}
$$

To facilitate the study of the dynamics of system (7), we define the following two isoclines:

$$
\begin{aligned}
& L_{1}: y=(1+p)(k-p), \\
& L_{2}: y=\frac{p}{a(1+p)} .
\end{aligned}
$$

\section{Poincaré Map and Its Main Properties}

We first construct the Poincare map to study the dynamics of system (7). According to the biological significance, this article only needs to be discussed in $\Omega$, where

$$
\Omega=\{(p, y) \mid p \geq 0, y \geq 0\} .
$$

Therefore, we first define the following two sets:

$$
\begin{aligned}
& S_{M}=\{(p, y) \mid p=\bar{p}, y \geq 0\}, \\
& S_{N}=\left\{(p, y) \mid p=\bar{p}+\frac{\tau}{1+\theta \bar{p}}, y \geq 0\right\} .
\end{aligned}
$$




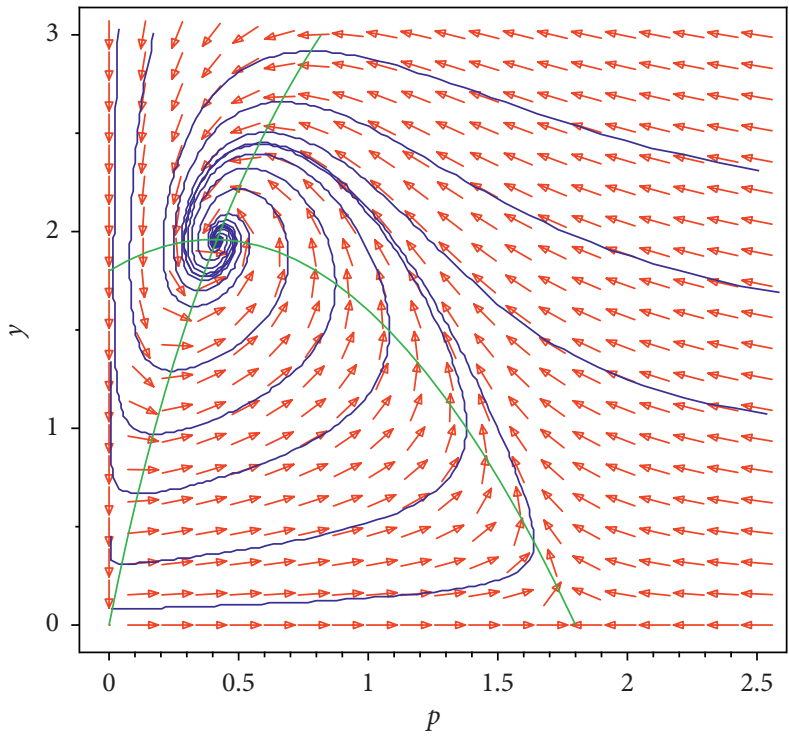

(a)

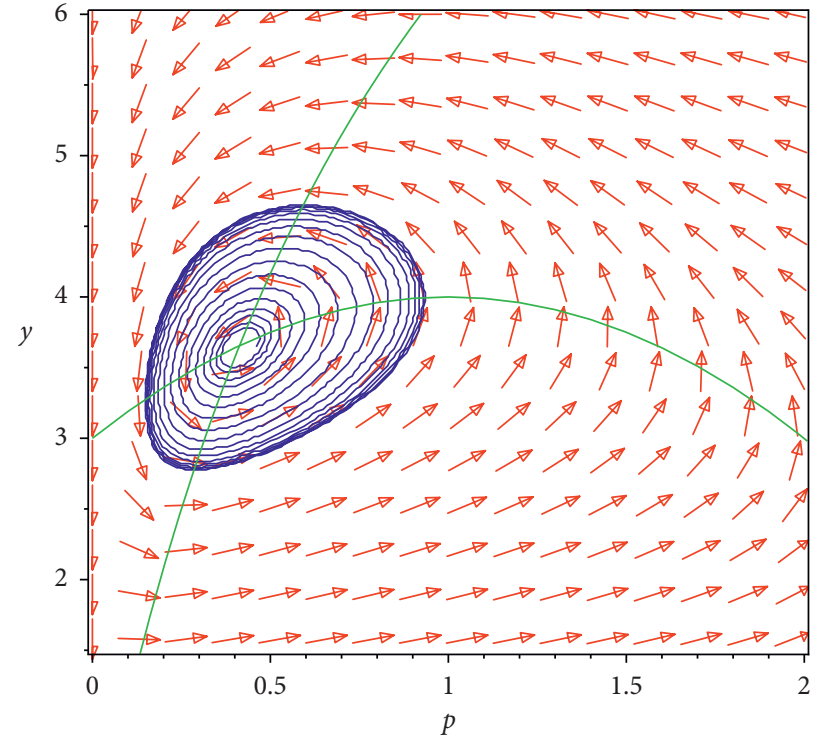

(b)

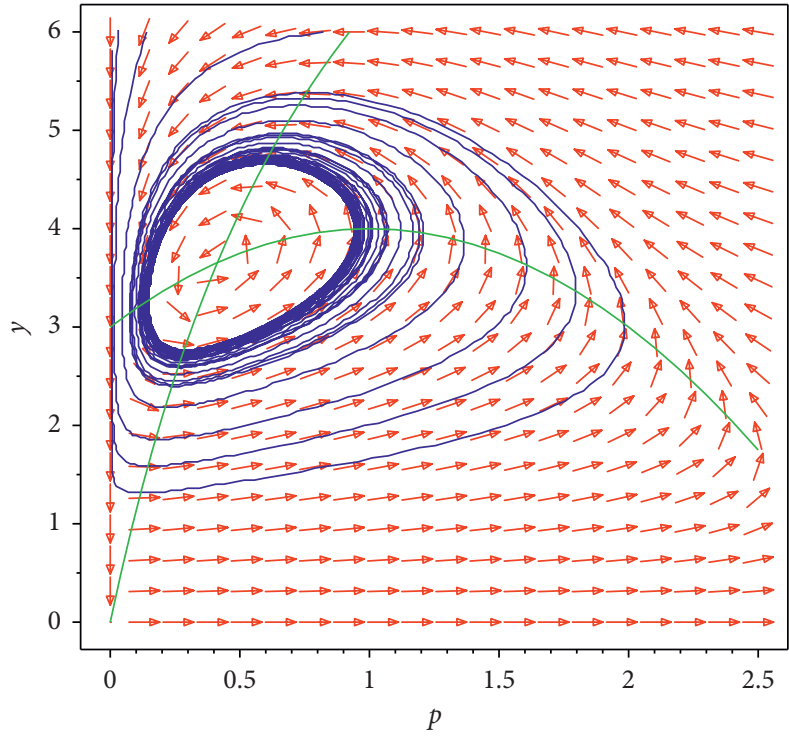

(c)

Figure 1: (a) Phase diagram of system (7) with $k=1.8$ and $a=0.15$. (b) The existence of the limit cycle with $k=3$ and $a=0.08$. (c) Stability of the limit cycle.

It is easy to know set $S_{M}$ and $S_{N}$ represents two parallel lines $L_{3}: p=\bar{p}$ and $L_{4}: p=\bar{p}+(\tau / 1+\theta \bar{p})$ in $\Omega$. We represent the intersection of $L_{4}$ and $L_{1}$ as $A\left(\bar{p}+(\tau / 1+\theta \bar{p}), y_{A}\right)$ and the intersection of $L_{3}$ and $L_{1}$ as $B\left(\bar{p}, y_{B}\right)$, where $y_{A}=$ $(1+\bar{p}+(\tau / 1+\theta \bar{p}))(k-\bar{p}-(\tau / 1+\theta \bar{p})) \quad$ and $y_{B}=(1+\bar{p})(k-\bar{p})$. And there exists a curve $\Gamma$ that starts at point $A$ and intersects with $L_{3}$ at point $C\left(\bar{p}, y_{C}\right)$.

According to the above definition, we construct the impulsive semidynamical system of system (7).

Define the impulsive set as

$$
M=\left\{(p, y) \mid p=\bar{p}, y \geq y_{C}\right\}
$$

while the continuous function uses the expression as follows:
$I:(\bar{p}, y) \in M \longrightarrow\left(p^{+}, y^{+}\right)=\left(\bar{p}+\frac{\tau}{1+\theta \bar{p}},\left(1-\frac{\delta y}{y+\beta}\right) y\right)$,

and the impulsive function $f(y)=(1-(\delta y / y+\beta)) y$ is increasing in $(0,+\infty)$. So, we represent the phase set as follows (Figure 2):

$$
\begin{aligned}
N= & I(M)=\left\{\left(p^{+}, y^{+}\right) \mid p^{+}=\bar{p}+\frac{\tau}{1+\theta \bar{p}}, y^{+}\right. \\
& \left.\geq\left(1-\frac{\delta y_{C}}{y_{C}+\beta}\right) y_{C}\right\} .
\end{aligned}
$$




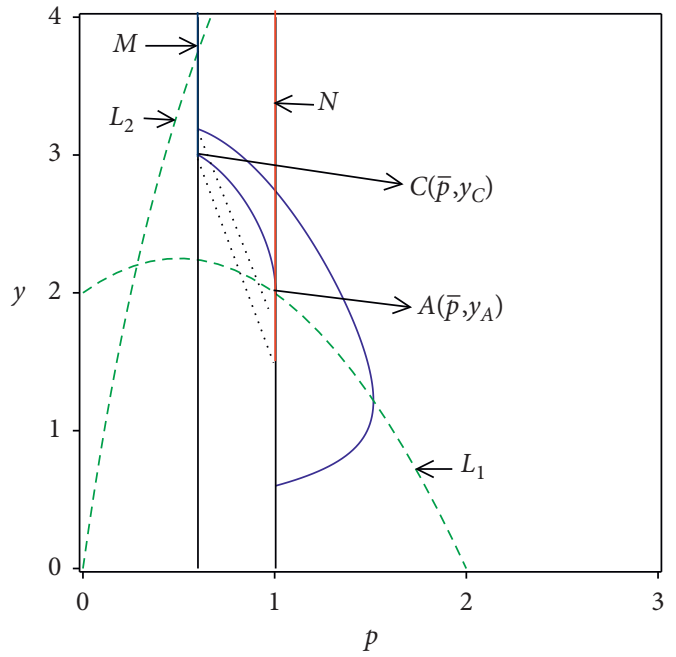

Figure 2: Phase set of system (7) with $k=2$ and $a=0.1$.

Unless otherwise specified, in this article, we assume that the initial point $A_{0}^{+}\left(p_{0}^{+}, y_{0}^{+}\right)$belongs to $N$. The trajectory through the point $A_{0}^{+}$is expressed as follows:

$$
\Pi\left(t, t_{0}, A_{0}^{+}\right)=\Pi\left(p\left(t, t_{0},\left(p_{0}^{+}, y_{0}^{+}\right)\right), y\left(t, t_{0},\left(p_{0}^{+}, y_{0}^{+}\right)\right)\right) .
$$

Next, we define the Poincaré map of system (6) in the case $\left(H_{1}\right)$.

Since the internal equilibrium point $D^{*}$ is the stable point, we assume that $\bar{p}>p^{*}$. Then, for any point $A_{k}^{+}=\left(\bar{p}+(\tau / 1+\theta \bar{p}), y_{k}^{+}\right) \in S_{N}$, the trajectory passing through point $A_{k}^{+}$will reach the impulse set $M$ through time $t_{1}$, so

$$
\begin{aligned}
& \Pi\left(t_{1}, \bar{p}+\frac{\tau}{1+\theta \bar{p}}, y_{k}^{+}\right) \\
& =\Pi\left(t_{1}, \bar{p}, y_{k+1}\right) \\
& =\Pi\left(p^{+}\left(t_{1}, \bar{p}+\frac{\tau}{1+\theta \bar{p}}, y_{k}^{+}\right), y^{+}\left(t_{1}, \bar{p}+\frac{\tau}{1+\theta \bar{p}}, y_{k}^{+}\right)\right) \\
& =\Pi\left(\bar{p}, y_{k+1}\right),
\end{aligned}
$$

where

$$
y_{k+1}=y^{+}\left(t_{1}, \bar{p}+\frac{\tau}{1+\theta \bar{p}}, y_{k}^{+}\right) .
$$

This indicates that the ordinate $y_{k+1}$ of the point at which the initial point on the phase set reaches the pulse set is determined by $y_{k}^{+}$. Then, we define $y_{k+1} \triangleq \varphi\left(y_{k}^{+}\right)$.

Therefore, the Poincaré map can be expressed as follows:

$$
\begin{aligned}
y_{k+1}^{+} & =\left(1-\frac{\delta y_{k+1}}{y_{k+1}+\beta}\right) y_{k+1}=\left(1-\frac{\delta \varphi\left(y_{k}^{+}\right)}{\varphi\left(y_{k}^{+}\right)+\beta}\right) \varphi\left(y_{k}^{+}\right) \\
& =F_{M}\left(y_{k}^{+}\right) .
\end{aligned}
$$

Then, system (8) is represented as the following scalar differential equation:

$$
\left\{\begin{array}{l}
\frac{\mathrm{d} y}{\mathrm{~d} p}=\frac{y((p / 1+p)-a y)}{p(k-p-(y / 1+p))} \triangleq l(p, y), \\
y\left(\bar{p}+\frac{\tau}{1+\theta \bar{p}}\right)=y_{0}^{+} .
\end{array}\right.
$$

Obviously, $l(p, y)$ is continuous and differentiable in $\Omega$. We make $p_{0}^{+}=\bar{p}+(\tau / 1+\theta \bar{p})$ and $y_{0}^{+}=\mu$ with $\mu \in(0,+\infty)$ for which $\left(p_{0}^{+}, y_{0}^{+}\right) \in \Omega$.

Define

$$
y(x)=y\left(x ; \bar{p}+\frac{\tau}{1+\theta \bar{p}}, \mu\right)=y(x, \mu), \bar{p} \leq x \leq \bar{p}+\frac{\tau}{1+\theta \bar{p}} .
$$

According to model (20), function $y(x, \mu)$ with initial value of $(\bar{p}+(\tau / 1+\theta \bar{p}), \mu)$ can be expressed as

$$
y(x, \mu)=\mu+\int_{x}^{\bar{p}+(\tau / 1+\theta \bar{p})} l(t, y(t, \mu)) \mathrm{d} t .
$$

Therefore, the form of the Poincare map is

$$
F_{M}(\mu)=y(\bar{p}, \mu)-\frac{\delta y^{2}(\bar{p}, \mu)}{y(\bar{p}, \mu)+\beta} .
$$

Theorem 2. We assume that system (8) has a stable internal equilibrium point which meets $k<2\left(p^{*}+1\right)$ and $\bar{p}>p^{*}$; then, the Poincaré map $F_{M}(\mu)$ of system (8) contains the following properties (Figure 3):

(i) The domain of $F_{M}(\mu)$ is $(0,+\infty)$

(ii) $F_{M}(\mu)$ is increasing on $\left[y_{A},+\infty\right)$ and decreasing on $\left(0, y_{A}\right]$

(iii) Poincaré map $F_{M}(\mu)$ is continuously differentiable

(iv) $F_{M}(\mu)$ has a unique fixed point

(v) $F_{M}(\mu)$ takes the minimum value at $y_{A}$ and has no maximum value

Proof.

(i) According to Theorem 1 and $\bar{p}>p^{*}$, for any point $A^{+}=(\bar{p}+(\tau / 1+\theta \bar{p}), \mu) \in S_{N}$, where $\mu \geq 0$ and the trajectory through point $A^{+}$always intersects the impulse set $M$. Therefore, the function $F_{M}(\mu)$ is meaningful when $\mu \in(0,+\infty)$. So, the domain of $F_{M}(\mu)$ is $(0,+\infty)$.

(ii) We first prove the monotonicity of the function $F_{M}(\mu)$ on the interval $\left[y_{A},+\infty\right)$. Choose any two points with $\mu_{k_{1}^{+}}, \mu_{k_{2}^{+}} \in\left[y_{A},+\infty\right)$, where $\mu_{k_{1}^{+}}<\mu_{k_{2}^{+}}$. Then, after time $t_{i}$, the trajectory $\Pi\left(t_{i}, \bar{p}+(\tau / 1+\right.$ $\left.\theta \bar{p}), \mu_{k_{i}^{+}}\right)=\Pi\left(t_{i}, \bar{p}, y_{k_{i}+1}\right) \in M, i=1,2, \quad$ where $y_{k_{i}+1} \in\left[y_{C},+\infty\right)$. From the properties of the solution of the differential equation, we get that 


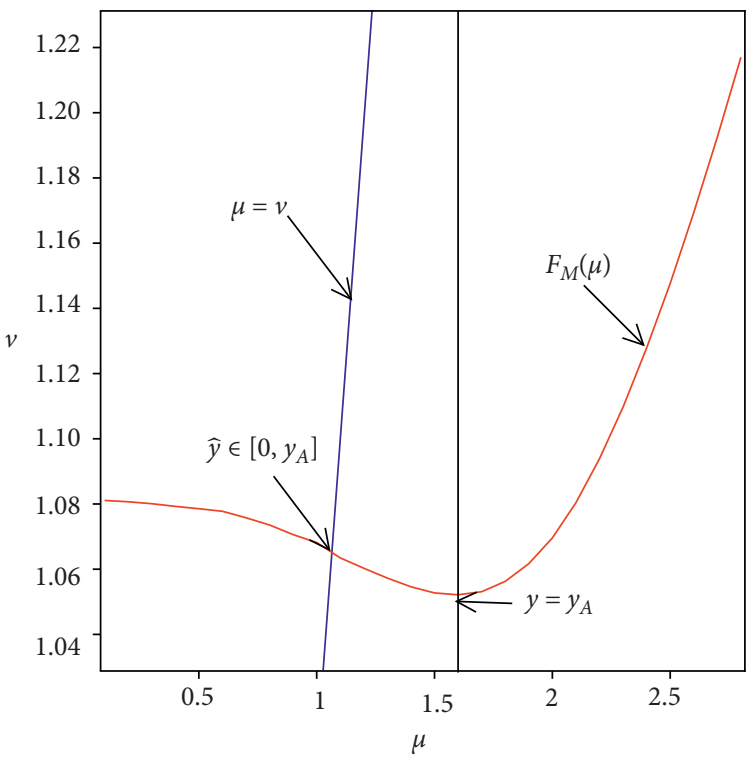

(a)

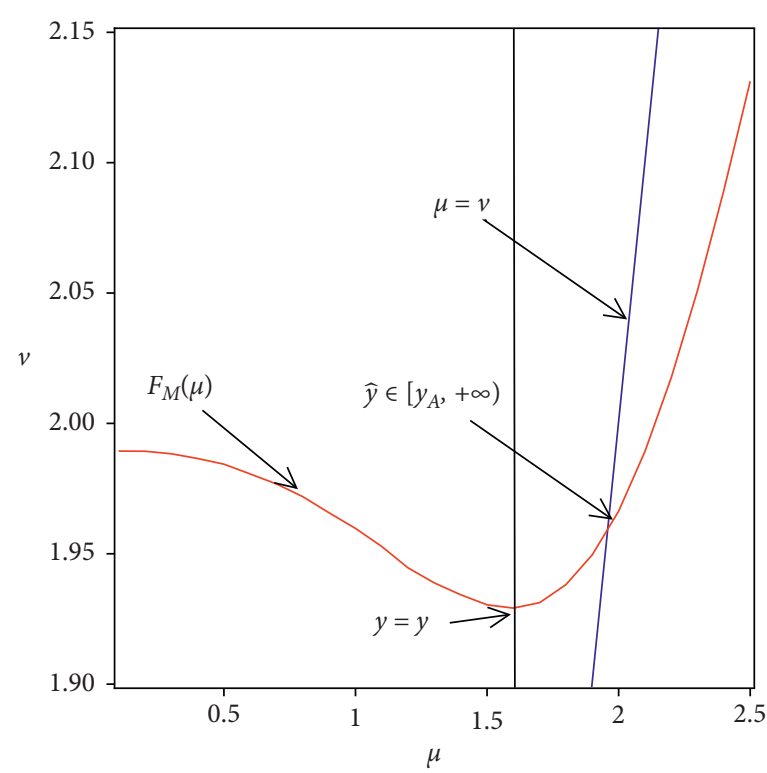

(b)

Figure 3: Image of Poincaré map $F_{M}(\mu)$ and the parameters fixed as $r=2.5, \varphi_{1}=0.2, a_{1}=0.3, \varphi_{2}=0.01, a_{2}=0.05$, and $E T=2.5$. (a) $b=0.7$. (b) $b=0.98$.

$y_{k_{1}+1}<y_{k_{2}+1}$; then, from system (19), the Poincaré map $F_{M}\left(\mu_{k_{1}^{+}}\right)<F_{M}\left(\mu_{k_{2}^{+}}\right)$. Therefore, $F_{M}(\mu)$ is increasing on $\left[y_{A},+\infty\right)$.

If $\mu_{k_{i}^{+}} \in\left(0, y_{A}\right]$ with $\mu_{k_{1}^{+}}<\mu_{k_{2}^{+}}$, the trajectory $\Pi(t, \bar{p}+$ $\left.(\tau / 1+\theta \bar{p}), \mu_{k_{i}^{+}}\right)$across the isocline $L_{1}$ and reach $S_{N}$ at points $A_{k_{1}^{\prime}}\left(\bar{p}+(\tau / 1+\theta \bar{p}), \mu_{k_{1}^{\prime}}\right)$ and $A_{k_{2}^{\prime}}\left(\bar{p}+(\tau / 1+\theta \bar{p}), \mu_{k_{2}^{\prime}}\right)$. From the Cauchy-Lipschitz theorem, we have $\mu_{k_{2}^{\prime}}>\mu_{k_{1}^{\prime}}$ and $\mu_{k_{i}^{\prime}} \in\left[y_{A},+\infty\right)$. According to the above proof, $F_{M}\left(\mu_{k_{1}^{+}}\right)$ $>F_{M}\left(\mu_{k_{2}^{+}}\right)$; therefore, $F_{M}(\mu)$ is decreasing on $\left(0, y_{A}\right]$.

(iii) From system (20), it is clear that $l(p, y)$ is continuous and differentiable in the first quadrant. According to the Cauchy-Lipschitz theorem and system (19), the function $F_{M}(\mu)$ is continuously differentiable in $R_{2}^{+}$.

(iv) First, we consider the value of the Poincare map $F_{M}(\mu)$ of $y_{A}$ and prove the existence of the fixed point. If $F_{M}\left(y_{A}\right)=y_{A}$, then the Poincaré map $F_{M}(\mu)$ possess a fixed point.

If $F_{M}\left(y_{A}\right)<y_{A}$, let $y_{q}^{+}=F_{M}\left(y_{A}\right) \in\left(0, y_{A}\right]$. From property (ii), we get when $y_{q}^{+}<y_{A}$, then $F_{M}\left(y_{q}^{+}\right)>F_{M}\left(y_{A}\right)=y_{q}^{+}$. In the light of property (iii) and the theorem of zero existence, there exists at least a point $\hat{y} \in\left(y_{q}^{+}, y_{A}\right)$, which meets $F_{M}(\hat{y})=\hat{y}$. From property (ii), $F_{M}(\mu)$ has at least a fixed point on $\left(0, y_{A}\right]$.

If $F_{M}\left(y_{A}\right)>y_{A}$, then $F_{M}\left(y_{A}\right)-y_{A}>0$. When $y_{0}^{+} \longrightarrow \infty$, from model (19), we obtain $y_{1}<y_{0}^{+}$and $y_{1}^{+}=y_{1}\left(1-\left(\delta y_{1} / y_{1}+\beta\right)\right)<y_{1}$. Then, $F_{M}\left(y_{0}^{+}\right)=y_{1}^{+}<y_{0}^{+}$. From the monotonicity and continuity of $F_{M}(\mu), F_{M}(\mu)$ has at least a fixed point $\hat{y} \in\left[y_{A},+\infty\right)$ in which $F_{M}(\hat{y})=\hat{y}$. Thus, $F_{M}(\mu)$ possess the fixed point on $(0,+\infty)$.
Next, the uniqueness of the fixed point is proved as follows. We assume that system (7) admits two fixed points $\hat{y}_{1}$ and $\hat{y}_{2}$, where $\hat{y}_{1}>\hat{y}_{2}$. Define $\Phi_{\hat{y}_{1} \hat{y}_{2}}(p)=\hat{y}_{1}(p)-\hat{y}_{2}(p)$. Since

$$
\begin{aligned}
& =\Phi_{\hat{y}_{1} \hat{y}_{2}}^{\prime}(p)=\hat{y}_{1}^{\prime}(p)-\hat{y}_{2}^{\prime}(p) \\
& =\frac{\hat{y}_{1}\left((p / 1+p)-a \hat{y}_{1}\right)}{p\left(k-p-\left(\hat{y}_{1} / 1+p\right)\right)}-\frac{\hat{y}_{2}\left((p / 1+p)-a \hat{y}_{2}\right)}{p\left(k-p-\left(\hat{y}_{2} / 1+p\right)\right)} \\
& =g^{\prime}(\xi)\left(\hat{y}_{1}-\hat{y}_{2}\right),
\end{aligned}
$$

where

$$
\begin{aligned}
g(y) & =\frac{y((p / 1+p)-a y)}{p(k-p-(y / 1+p))} \\
g^{\prime}(y) & =\frac{\left(p\left(k p-p^{2}+a y^{2}\right) / 1+p\right)+2 a y p(p-k)}{[p(k-p-(y / 1+p))]^{2}},
\end{aligned}
$$

therefore, when $k<\left(p^{2}-a y^{2} / p\right)$, we have $g^{\prime}(y)<0$. Then, $\Phi_{\hat{y}_{1} \hat{y}_{2}}^{\prime}(p)<0$ which means that $\Phi_{\widehat{y}_{1} \hat{y}_{2}}^{\prime}(p)$ is a monotonically decreasing function and $\Phi_{\widehat{y}_{1} \hat{y}_{2}}(\bar{p}+(\tau / 1+\theta \bar{p}))<\Phi_{\hat{y}_{1} \hat{y}_{2}}(\bar{p})$. Then,

$$
\begin{aligned}
\beta= & \frac{\delta \hat{y}_{1}^{2}}{\hat{y}_{1}-\widehat{y}_{1}^{+}}-\hat{y}_{1} \\
= & \frac{\delta\left[\hat{y}_{2}+\Phi_{\hat{y}_{1} \hat{y}_{2}}(\bar{p}+(\tau / 1+\theta \bar{p}))\right]^{2}}{\hat{y}_{2}+\Phi_{\hat{y}_{1} \hat{y}_{2}}(\bar{p}+(\tau / 1+\theta \bar{p}))-\left[\hat{y}_{2}+\Phi_{\hat{y}_{1} \hat{y}_{2}}(\bar{p})\right]} \\
& -\left[\widehat{y}_{2}+\Phi_{\hat{y}_{1} \hat{y}_{2}}(\bar{p}+(\tau / 1+\theta \bar{p}))\right] \\
> & \frac{\delta \widehat{y}_{2}^{2}}{\hat{y}_{2}-\hat{y}_{2}^{+}}-\hat{y}_{2}=\beta,
\end{aligned}
$$


which leads to a contradiction. Thus, the fixed point of system (7) is unique when $k<\left(p^{2}-a y^{2} / p\right)$.

(v) From property (ii), we obtain that $F_{M}(\mu)$ is decreasing on $\left(0, y_{A}\right]$ and increasing on $\left[y_{A},+\infty\right)$. Thus, for any $\mu \in(0,+\infty)$, there is $F_{M}(\mu) \geq F_{M}\left(y_{A}\right)$. So, when $\mu=y_{A}$, the function $F_{M}(\mu)$ reaches the minimal value which is also the minimum value. And the minimum value is $F_{M}\left(y_{A}\right)=$ $y\left(\bar{p}, y_{A}\right)-\delta y^{2}\left(\bar{p}, y_{A}\right) / y\left(\bar{p}, y_{A}\right)+\beta$.

Since $F_{M}(\mu)$ is increasing on $\left[y_{A},+\infty\right)$, then $F_{M}(\mu)$ has only a minimum value on $(0,+\infty)$ and no maximum value.

\section{Periodic Solution of the System}

5.1. Boundary Periodic Solution. In the absence of herbivores and $\delta=0$, system (7) possess the boundary periodic solution. Thus, we represent the system as

$$
\left\{\begin{array}{l}
\frac{\mathrm{d} p(t)}{\mathrm{d} t}=p(t)(k-p(t)) p(t)>\bar{p} \\
p\left(t^{+}\right)=p(t)+\frac{\tau}{1+\theta p(t)}, p(t)=\bar{p}
\end{array}\right.
$$

where the initial condition is $p\left(0^{+}\right)=\bar{p}+(\tau / 1+\theta \bar{p})$, and by calculating the first equation, we have

$$
p(t)=\frac{[(1+\theta \bar{p}) \bar{p}+\tau] k}{[(1+\theta \bar{p}) \bar{p}-k \theta \bar{p}+\tau-k] \exp (-k t)-(1+\theta \bar{p}) \bar{p}-\tau} .
$$

Supposing the solution starting from $(p(t), 0)$ reaches the line $L_{3}$ at time $T$, one has

$$
\bar{p}=\frac{[(1+\theta \bar{p}) \bar{p}+\tau] k}{[(1+\theta \bar{p}) \bar{p}-k \theta \bar{p}+\tau-k] \exp (-k T)-(1+\theta \bar{p}) \bar{p}-\tau} .
$$

Solving the above equation on $T$, then

$$
T=-\frac{1}{k} \ln \left[-\frac{\theta k \bar{p}^{2}-\theta \bar{p}^{3}+k \tau-\bar{p} \tau+k \bar{p}-\bar{p}^{2}}{\bar{p}\left(-k \theta \bar{p}+\theta \bar{p}^{2}+\tau-k+\bar{p}\right)}\right] \text {. }
$$

Thus, the boundary periodic solution $\left(p^{T}(t), 0\right)$ of model (7) with period $T$ is

$$
p^{T}(t)=\frac{[(1+\theta \bar{p}) \bar{p}+\tau] k}{[(1+\theta \bar{p}) \bar{p}-k \theta \bar{p}+\tau-k] \exp (-k t)-(1+\theta \bar{p}) \bar{p}-\tau} \text {. }
$$

Theorem 3. When condition $\left|\chi_{1}\right|<1$ is satisfied, then the boundary periodic solution $\left(p^{T}(t), 0\right)$ is orbitally asymptotically stable, and

$$
\chi_{1}=\Delta_{1} \exp \left(\int_{0}^{T} Q(t) \mathrm{d} t\right)
$$

where

$$
\Delta_{1}=\frac{(\bar{p}+(\tau / 1+\theta \bar{p}))(k-\bar{p}-(\tau / 1+\theta \bar{p}))\left[1-\left(\delta y^{2}+2 \beta \delta y /(y+\beta)^{2}\right)\right]}{\bar{p}(k-\bar{p})} .
$$

Proof. Let $F(p, y)=p(k-p-(y / 1+p)), G(p, y)=y((p /$ $1+p)-a y), \quad \alpha(p, y)=\tau /(1+\theta p), \quad \beta(p, y)=-\delta y^{2} / y+\beta$, and $\Phi(p, y)=p-\bar{p},\left(p^{T}(T), y^{T}(T)\right)=(\bar{p}, 0), \quad\left(p^{T}\left(T^{+}\right)\right.$, $\left.y^{T}\left(T^{+}\right)\right)=(\bar{p}+(\tau / 1+\theta \bar{p}), 0)$.

Then,

$$
\begin{aligned}
\frac{\partial F}{\partial p} & =k-2 p-\frac{y}{(1+p)^{2}}, \frac{\partial G}{\partial y}=\frac{p}{1+p}-2 a y, \frac{\partial \alpha}{\partial p}=\frac{-\tau \theta}{(1+\theta p)^{2}}, \\
\frac{\partial \beta}{\partial y} & =-\frac{\delta y^{2}+2 \beta \delta y}{(y+\beta)^{2}}, \frac{\partial \Phi}{\partial p}=1, \frac{\partial \alpha}{\partial y}=\frac{\partial \beta}{\partial p}=\frac{\partial \Phi}{\partial y}=0 . \\
\Delta_{1} & =\frac{F_{+}((\partial \beta / \partial y)(\partial \Phi / \partial p)-(\partial \beta / \partial p)(\partial \Phi / \partial y)+(\partial \Phi / \partial p))+G_{+}((\partial \alpha / \partial p)(\partial \Phi / \partial y)-(\partial \alpha / \partial y)(\partial \Phi / \partial p)+(\partial \Phi / \partial y))}{F(\partial \Phi / \partial p)+G(\partial \Phi / \partial y)} \\
& =\frac{\left.F_{+}(\bar{p}+(\tau / 1+\theta \bar{p}), 0)\right)(1+(\partial \beta / \partial g))}{F(\bar{p}, 0)} \\
& =\frac{(\bar{p}+(\tau / 1+\theta \bar{p}))(k-\bar{p}-(\tau / 1+\theta \bar{p}))\left[1-\left(\delta y^{2}+2 \beta \delta y /(y+\beta)^{2}\right)\right]}{\bar{p}(k-\bar{p})} \\
& \int_{0}^{T}\left[\frac{\partial F}{\partial p}\left(p^{T}(T), y^{T}(T)\right)+\frac{\partial G}{\partial y}\left(p^{T}(T), y^{T}(T)\right)\right] \mathrm{d} t=\int_{0}^{T}\left[k-2 p^{T}(t)+\frac{p^{T}(t)}{1+p^{T}(t)}\right] \mathrm{d} t \\
& =\int_{0}^{T} Q(t) \mathrm{d} t .
\end{aligned}
$$


Furthermore,

$$
\begin{aligned}
\chi_{1} & =\Delta_{1} \exp \left(\int_{0}^{T}\left[\frac{\partial F}{\partial p}\left(p^{T}(T), y^{T}(T)\right)+\frac{\partial G}{\partial g}\left(p^{T}(T), y^{T}(T)\right)\right] \mathrm{d} t\right) \\
& =\Delta_{1} \exp \left(\int_{0}^{T} Q(t) \mathrm{d} t\right) .
\end{aligned}
$$

Therefore, from Lemma 1, we obtain the conclusion of Theorem 3 when $\left|\chi_{1}\right|<1$.

5.2. Order-k Periodic Solution and Its Stability Analysis. According to Theorem 2 (iv), we conclude that system (7) has a unique fixed point, which implies that the system possesses an order-1 periodic solution. In this section, we prove the stability of periodic solution and the existence of order- $k(k \geq 2)$ periodic solution. So, we make the following provisions.

For $A_{0}^{+}\left(\bar{p}+(\tau / 1+\theta \bar{p}), y_{0}^{+}\right) \in N$, the trajectory of the initial point $A_{0}^{+}$will reach the impulse set $M$, which is applied to the point $A_{1}^{+}\left(\bar{p}+(\tau / 1+\theta \bar{p}), y_{1}^{+}\right)$by the impulse. In the light of the definition of the Poincare map, we have $F_{M}\left(y_{0}^{+}\right)=y_{1}^{+}$, and repeating the process above, then

$$
F_{M}\left(y_{1}^{+}\right)=F_{M}\left[F_{M}\left(y_{0}^{+}\right)\right] \triangleq F_{M}^{2}\left(y_{0}^{+}\right)=y_{2}^{+} .
$$

Further promotion,

$$
y_{k}^{+}=F_{M}^{k}\left(y_{0}^{+}\right) .
$$

The sequence $\left\{y_{k}^{+}\right\}$is available, $k=0,1,2, \ldots$

Theorem 4. The order-1 period solution $(\xi(t), \eta(t))$ is orbitally asymptotically stable if

$$
\left|\frac{(\bar{p}+(\tau / 1+\theta \bar{p}))\left(k-\bar{p}-(\tau / 1+\theta \bar{p})-\left(\eta_{0} / 1+\bar{p}+(\tau / 1+\theta \bar{p})\right)\right)\left[1-\left(\delta y^{2}+2 \beta \delta y /(y+\beta)^{2}\right)\right]}{\bar{p}\left(k-\bar{p}-\left(\eta_{0} / 1+\bar{p}\right)\right)} \exp \left(\int_{0}^{T} U(t) \mathrm{d} t\right)\right|<1
$$

where

$$
U(t)=\left(k-2 p-\frac{y}{(1+p)^{2}}+\frac{p}{1+p}-2 a y\right)
$$

Proof. We define the starting and the ending point of the order-1 periodic solution as $(\xi(T), \eta(T))=\left(\bar{p}, \eta_{0}\right)$ and $\left(\xi\left(T^{+}\right), \eta\left(T^{+}\right)\right)=\left(\bar{p}+(\tau / 1+\theta \bar{p}), \eta_{0}-\left(\delta \eta_{0}^{2} / \eta_{0}+\beta\right)\right)$, respectively.

Thus,

$$
\begin{aligned}
\Delta_{2} & =\frac{F_{+}\left(\bar{p}+(\tau / 1+\theta \bar{p}), \eta_{0}-\left(\delta \eta_{0}^{2} / \eta_{0}+\beta\right)\right)\left[1-\left(\delta y^{2}+2 \beta \delta y /(y+\beta)^{2}\right)\right]}{F\left(\bar{p}, \eta_{0}\right)} \\
& =\frac{(\bar{p}+(\tau / 1+\theta \bar{p}))\left(k-\bar{p}-(\tau / 1+\theta \bar{p})-\left(\eta_{0} / 1+\bar{p}+(\tau / 1+\theta \bar{p})\right)\right)\left[1-\left(\delta y^{2}+2 \beta \delta y /(y+\beta)^{2}\right)\right]}{\bar{p}\left(k-\bar{p}-\left(\eta_{0} / 1+\bar{p}\right)\right)} \\
\int_{0}^{T}\left(\frac{\partial F}{\partial p}+\frac{\partial G}{\partial y}\right) \mathrm{d} t & =\int_{0}^{T}\left(k-2 p-\left(y /(1+p)^{2}\right)+(p / 1+p)-2 a y\right) \mathrm{d} t=\int_{0}^{T} U(t) \mathrm{d} t .
\end{aligned}
$$

The multiplier $\chi_{2}$ is obtained:

$$
\chi_{2}=\Delta_{2} \exp \left(\int_{0}^{T} U(t) \mathrm{d} t\right)
$$

From (38), we obtain $\left|\chi_{2}\right|<1$; thus, the order-1 periodic solution is locally stable.

Theorem 5. When conditions $\left(H_{1}\right)$ and $F_{M}\left(y_{A}\right)>y_{A}$ are established, then the order-1 periodic solution of system (7) is globally asymptotic stable.

Proof. According to Theorem 2, when $F_{M}\left(y_{A}\right)>y_{A}$, system (7) possesses unique fixed point $\hat{y}$, which means system (7) has an order- 1 periodic solution, where $\hat{y} \in\left[y_{A},+\infty\right)$. Also, we get the Poincaré map $F_{M}(\mu)$ which increases on $\left[y_{A},+\infty\right)$.
For any trajectory in which the initial point is $A_{0}^{+}\left(\bar{p}+(\tau / 1+\theta \bar{p}), y_{0}^{+}\right)$, when $y_{A}<y_{0}^{+}<\hat{y}$, since $F_{M}(\mu)$ is increasing on $\left[y_{A},+\infty\right)$ and there exists a unique fixed point, then $y_{A}<F_{M}\left(y_{A}\right)<F_{M}\left(y_{0}^{+}\right)=y_{1}^{+}<F_{M}(\widehat{y})=\widehat{y}$ and $y_{1}^{+}<$ $F_{M}\left(y_{1}^{+}\right)=y_{2}^{+}<\widehat{y}$. It can be inferred from this that $y_{A}<F_{M}\left(y_{0}^{+}\right)<\ldots<F_{M}^{n-1}\left(y_{0}^{+}\right)<F_{M}^{n}\left(y_{0}^{+}\right)<\ldots<\hat{y}$. According to the monotonically bounded sequence, we get $\lim _{n \longrightarrow+\infty} F_{M}^{n}\left(y_{0}^{+}\right)=\hat{y}$.

When $\hat{y}<y_{0}^{+}$, by using a similar approach, we have $\hat{y}=$ $F_{M}(\widehat{y})<F_{M}\left(y_{0}^{+}\right)<y_{0}^{+}$and $F_{M}\left(y_{1}^{+}\right)=y_{2}^{+}<y_{1}^{+}=F_{M}\left(y_{0}^{+}\right)$. Furthermore, we get $\hat{y}<\ldots<F_{M}^{n}\left(y_{0}^{+}\right)<F_{M}^{n-1}\left(y_{0}^{+}\right)<\ldots<$ $y_{0}^{+}$. Then, $\lim _{n \longrightarrow+\infty} F_{M}^{n}\left(y_{0}^{+}\right)=\hat{y}$. Therefore, the order-1 periodic solution of system (7) is globally asymptotic stable.

Theorem 6. If conditions $\left(H_{1}\right), \quad F_{M}\left(y_{A}\right)<y_{A}$, and $F_{M}^{2}\left(y_{A}\right)<y_{A}$ are established, then model (7) has a stable order-1 or order-2 periodic solution. 
Proof. For any point $A_{0}^{+}\left(\bar{p}+(\tau / 1+\theta \bar{p}), y_{0}^{+}\right) \in N$, where $y_{0}^{+} \in(0,+\infty)$, we discuss the following two cases of function $F_{M}\left(y_{0}^{+}\right)$which are defined by point $y_{0}^{+}$.

(i) When $y_{0}^{+} \in\left[y_{A},+\infty\right)$, since the function $F_{M}(\mu)$ monotonically increases and has no fixed point when $\mu \in\left[y_{A},+\infty\right)$, therefore, there must be a positive integer $m$ so that $y_{m-1}^{+}=F_{M}\left(y_{m-2}^{+}\right)>y_{A}$ and $F_{M}\left(y_{m-1}^{+}\right)=y_{m}^{+}<y_{A} ; \quad$ then, $y_{m}^{+}=F_{M}\left(y_{m-1}^{+}\right)>$ $F_{M}\left(y_{A}\right)$ and $y_{m}^{+} \in\left[F_{M}\left(y_{A}\right), y_{A}\right]$

(ii) When $y_{0}^{+} \in\left(0, y_{A}\right]$, since the function $F_{M}(\mu)$ which decreases in $\left(0, y_{A}\right]$ and $F_{M}^{2}\left(y_{A}\right)<y_{A}$ and $F_{M}\left(y_{A}\right)<y_{\underline{A}}$, there must exist $\bar{m}$ which satisfies $F_{M}\left(y_{A}\right)<F_{M}^{\bar{m}}\left(y_{0}^{+}\right)<y_{A}$, where $\bar{m} \geq 1$

Through the above discussion, when $y_{0}^{+} \in(0,+\infty)$, there always exist a positive integer $m$ which satisfies $F_{M}\left(y_{A}\right)<F_{M}^{m}\left(y_{0}\right)<y_{A}$. Since $F_{M}(\mu)$ is decreasing in $\left[F_{M}\left(y_{A}\right), y_{A}\right]$, thus,

$$
F_{M}\left(\left[F_{M}\left(y_{A}\right), y_{A}\right]\right)=\left[F_{M}\left(y_{A}\right), F_{M}^{2}\left(y_{A}\right)\right] \subset\left[F_{M}\left(y_{A}\right), y_{A}\right] \text {. }
$$

So, we only need to discuss the case of function $F_{M}(\mu)$ in interval $\left[F_{M}\left(y_{A}\right), y_{A}\right] \cdot A_{0}^{+}\left(\bar{p}+(\tau / 1+\theta \bar{p}), y_{0}^{+}\right)$is the trajectory of the initial point, where $y_{0}^{+} \in\left[F_{M}\left(y_{A}\right), y_{A}\right]$. After the action of the impulse, we get the sequence $y_{n}^{+}, n=0,1,2 \ldots$, where $y_{n}^{+}=F_{M}^{n}\left(y_{0}^{+}\right), n=1,2 \ldots$.

According to the magnitude of $y_{n}^{+}$, there exist four situations:

Situation 1. $y_{A} \geq F_{M}\left(y_{0}^{+}\right)>y_{0}^{+}>F_{M}\left(y_{1}^{+}\right) \geq F_{M}\left(y_{A}\right)$ (Figure 4(a)); then, $y_{A} \geq y_{1}^{+}>y_{0}^{+}>y_{2}^{+}>F_{M}\left(y_{A}\right)$, and thus, we have $y_{3}^{+}=F_{M}\left(y_{2}^{+}\right)>F_{M}\left(y_{0}^{+}\right)=y_{1}^{+}$and $y_{4}^{+}=F_{M}\left(y_{3}^{+}\right)<F_{M}\left(y_{1}^{+}\right)=$ $y_{2}^{+}$, so $y_{3}^{+}>y_{1}^{+}>y_{0}^{+}>y_{2}^{+}>y_{4}^{+}$. After an infinite number of pulses, we get

$$
\begin{aligned}
y_{A} & \geq \ldots \geq y_{2 n+1}^{+}>y_{2 n-1}^{+}>\ldots>y_{1}^{+}>y_{0}^{+}>y_{2}^{+}>\ldots \\
& >y_{2 n}^{+}>y_{2 n+2}^{+}>\ldots>F_{M}\left(y_{A}\right)
\end{aligned}
$$

Situation 2. $y_{A} \geq F_{M}\left(y_{0}^{+}\right)>F_{M}\left(y_{1}^{+}\right)>y_{0}^{+} \geq F_{M}\left(y_{A}\right)$ (Figure 4(b)); furthermore, we have $F_{M}\left(y_{1}^{+}\right)=y_{2}^{+}<F_{M}\left(y_{2}^{+}\right)=$ $y_{3}^{+}<F_{M}\left(y_{0}^{+}\right)=y_{1}^{+}$and $F_{M}\left(y_{3}^{+}\right)=y_{4}^{+}>F_{M}\left(y_{1}^{+}\right)=y_{2}^{+}$. After an infinite number of pulses, we get

$$
\begin{aligned}
y_{A} & \geq y_{1}^{+}>\ldots \geq y_{2 n-1}^{+}>y_{2 n+1}^{+}>\ldots>y_{2 n+2}^{+}>y_{2 n}^{+}>\ldots \\
& >y_{2}^{+}>y_{0}^{+}>F_{M}\left(y_{A}\right)
\end{aligned} .
$$

Situation 3. $y_{A}>y_{0}^{+}>F_{M}\left(y_{1}^{+}\right)>F_{M}\left(y_{0}^{+}\right) \geq F_{M}\left(y_{A}\right)$; from the above similar methods, we obtain

$$
\begin{aligned}
y_{A} & >y_{0}^{+}>y_{2}^{+}>\ldots>y_{2 n}^{+}>y_{2 n+2}^{+}>\ldots>y_{2 n+1}^{+}>y_{2 n-1}^{+} \\
& >\ldots>y_{1}^{+} \geq F_{M}\left(y_{A}\right) .
\end{aligned}
$$

Situation 4. $y_{A}>F_{M}\left(y_{1}^{+}\right)>y_{0}^{+}>F_{M}\left(y_{0}^{+}\right) \geq F_{M}\left(y_{A}\right)$; from the above similar methods, we get

$$
\begin{aligned}
y_{A} & \geq \ldots>y_{2 n+2}^{+}>y_{2 n}^{+}>\ldots>y_{2}^{+}>y_{0}^{+}>y_{1}^{+}>\ldots \\
& >y_{2 n-1}^{+}>y_{2 n+1}^{+}>\ldots \geq F_{M}\left(y_{A}\right)
\end{aligned}
$$

For situations 2 and 3 , sequences $\left\{y_{2 n}^{+}\right\}$and $\left\{y_{2 n+1}^{+}\right\}$are monotonically bounded. Then, we have $\lim _{n \longrightarrow \infty} y_{2 n}^{+}=\lim _{n \longrightarrow \infty} y_{2 n-1}^{+}$or $\lim _{n \longrightarrow \infty} n \longrightarrow \infty y_{2 n}^{+}=\widehat{y}_{1}$ and $\lim _{n \longrightarrow \infty} y_{2 n-1}^{+}=\widehat{y}_{2}$, where $\widehat{y}_{1} \neq \widehat{y}_{2}$. This indicates that, in situations 2 and 3, system (7) has stable order-1 or order-2 periodic solution.

From situations 1 and $4, \lim _{n \longrightarrow \infty} y_{2 n+1}^{+}=y_{1}^{*} \leq y_{A}$ and $\lim _{n \longrightarrow \infty} y_{2 n-1}^{+}=y_{2}^{*} \geq F_{M}\left(y_{A}\right)$. Therefore, in situations 1 and 4 , system (7) has stable order-2 periodic solution.

Theorem 6 shows that system (7) has stable order-1 or order-2 periodic solutions under certain conditions. However, this theorem does not give sufficient and necessary conditions for the global stability of order-1 periodic solution. Therefore, Theorem 7 is given as follows:

Theorem 7. When $F_{M}\left(y_{A}\right)<y_{A}$, then the sufficient and necessary conditions for the global asymptotic stability of the order-1 periodic solution of system (7) are $F_{M}^{2}\left(y_{0}^{+}\right)<y_{0}^{+}$for any $y_{0}^{+} \in\left[\hat{y}, y_{A}\right]$, where $F_{M}(\widehat{y})=\widehat{y}$.

Proof. From Theorem 2, a stable order-1 periodic solution is available in system (7) when $F_{M}\left(y_{A}\right)<y_{A}$. Then, system (7) has $\hat{y} \in\left[F_{M}\left(y_{A}\right), y_{A}\right]$ in which $F_{M}(\hat{y})=\hat{y}$. For any $y_{0}^{+} \in\left(\hat{y}, y_{A}\right]$, make $y_{n}^{+}=F_{M}^{n}\left(y_{0}^{+}\right), n=1,2, \ldots$, and according to the properties of Poincare map $F_{M}(\mu)$, we have $F_{M}\left(y_{A}\right)<y_{1}^{+}<\hat{y}<y_{2}^{+}<y_{0}^{+}<y_{A} ;$ furthermore, we get $F_{M}\left(y_{A}\right)<y_{2 n+1}^{+}<\hat{y}<y_{2 n}^{+}<y_{A}$. From the monotone boundedness of the sequence, we know $\lim _{n \rightarrow \infty} y_{2 n}^{+}=$ $\lim _{n \longrightarrow \infty} y_{2 n+1}^{+}=\hat{y}$. Therefore, the order-1 periodic solution of system (7) is globally asymptotically stable.

We assume the order-1 periodic solution is globally stable, and the following proves that for any $y_{0}^{+} \in\left(\hat{y}, y_{A}\right]$ satisfies $F_{M}^{2}\left(y_{0}^{+}\right)<y_{0}^{+}$. If this condition is not met, then there exists minimum $y_{0}^{+} \in\left(\hat{y}, y_{A}\right]$ which makes $F_{M}^{2}\left(y_{0}^{+}\right) \geq y_{0}^{+}$. From Theorem 6 , we get that, for any small $\varepsilon>0$, there exists $y_{1}^{+} \in(\widehat{y}-\varepsilon, \widehat{y})$ which makes $F_{M}^{2}\left(y_{1}^{+}\right)<y_{1}^{+}$. From the continuity of $F_{M}(\mu)$, there exists $\widehat{y} \in\left(y_{1}^{+}, y_{0}^{+}\right)$which makes $F_{M}^{2}(\widehat{\hat{y}})=\widehat{\hat{y}}$. Therefore, system (7) has order-2 periodic solution, which is contradictory to the global stability of order1 periodic solution.

Theorem 8. When $F_{M}\left(y_{A}\right)<y_{A}$ and $F_{M}^{2}\left(y_{A}\right)<y_{m}^{+}$, where $F_{M}\left(y_{m}^{+}\right)=y_{A}, y_{m}^{+}=\min \left\{y^{+}, F_{M}\left(y^{+}\right)=y_{A}\right\}$, then system (7) has the order-3 periodic solution.

Proof. Since $F_{M}\left(y_{A}\right)<y_{A}$, from Theorem 2, we get that system (7) has unique $\hat{y} \in\left(F_{M}\left(y_{A}\right), y_{A}\right)$, which makes $F_{M}(\hat{y})=\hat{y}$. Since Poincaré map $F_{M}(\mu)$ is continuous in closed intervals $[0, \hat{y}], F_{M}(0)>0$, and $F_{M}(\hat{y})=\hat{y}$, from the intermediate value theorem, there is $y_{m}^{+} \in(0, \hat{y})$ which makes $F_{M}\left(y_{m}^{+}\right)=y_{A}$, and then $F_{M}^{3}\left(y_{m}^{+}\right)=F_{M}^{2}\left(y_{A}\right)<y_{m}^{+}$. Thus, we have $F_{M}^{3}\left(y_{m}^{+}\right)-y_{m}^{+}<0$. 


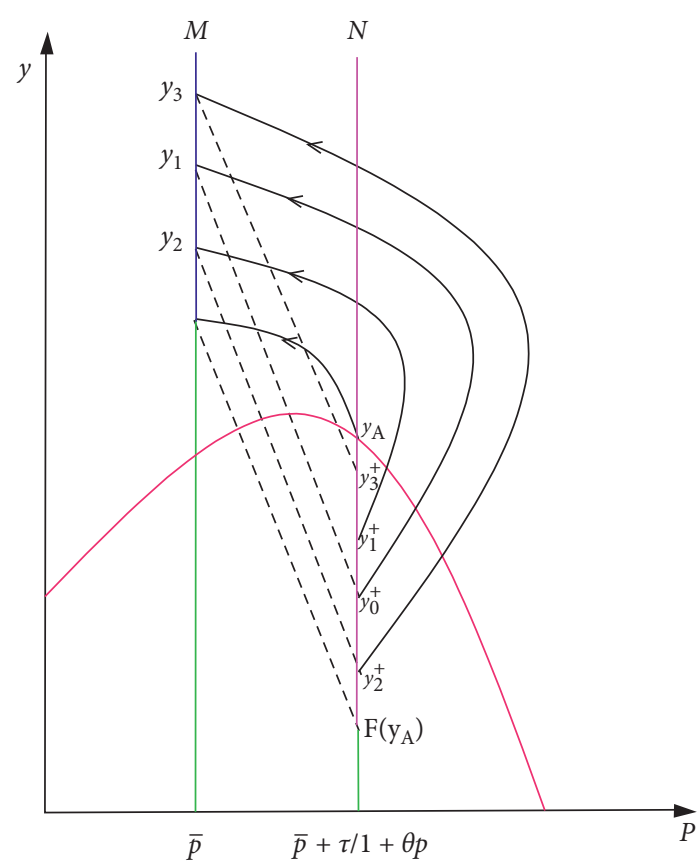

(a)

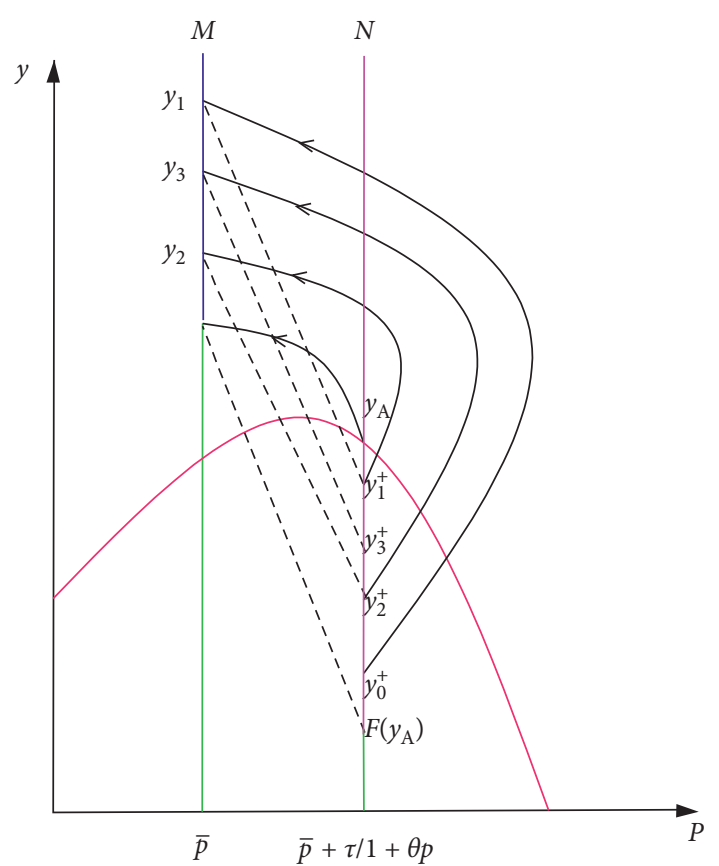

(b)

Figure 4: Path curve of system (7). (a) $y_{A} \geq y_{1}^{+}>y_{0}^{+}>y_{2}^{+} \geq F_{M}\left(y_{A}\right)$. (b) $y_{A} \geq y_{1}^{+}>y_{2}^{+}>y_{0}^{+} \geq F_{M}\left(g_{A}\right)$.

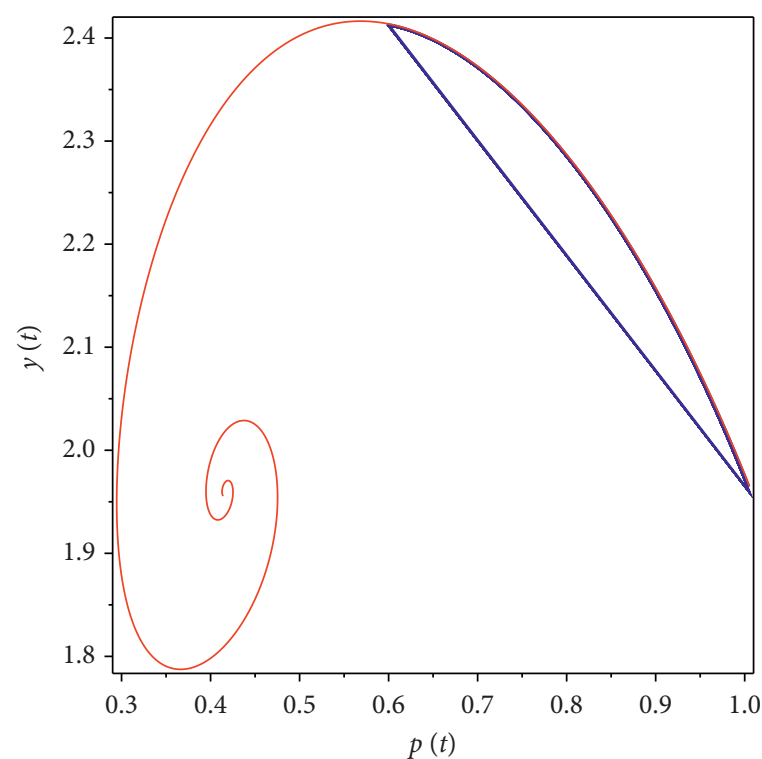

(a)

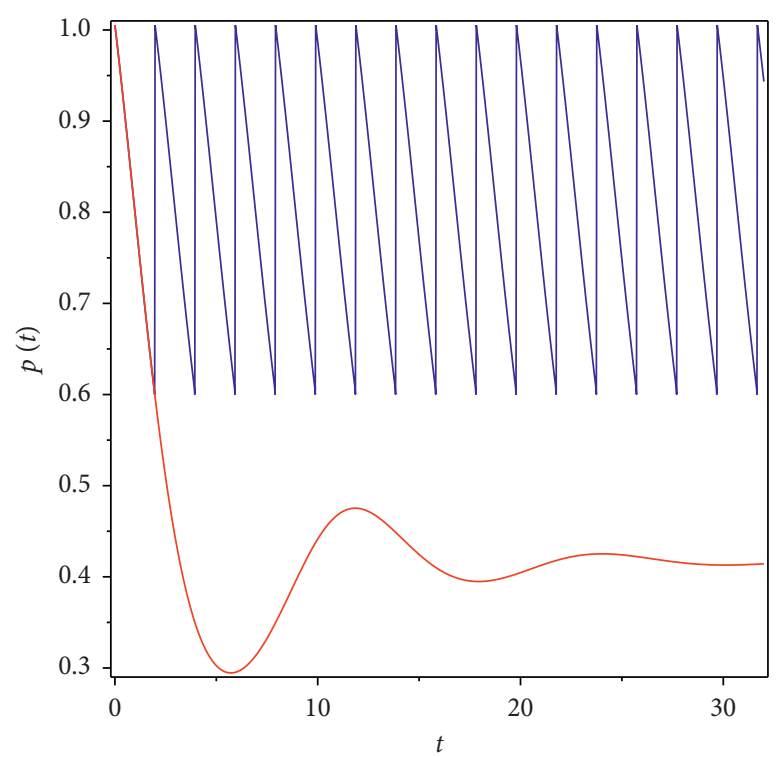

(b)

Figure 5: Continued. 


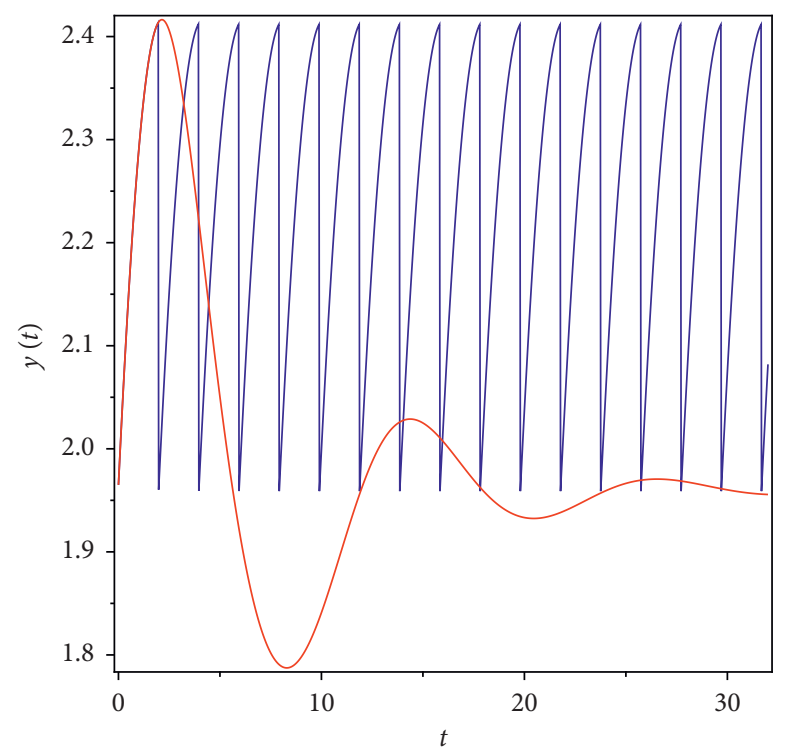

(c)

Figure 5: Phase portrait and time series of system $k=1.8, a=0.15, \tau=0.6, \bar{p}=0.6, \theta=0.8, \delta=0.25$, and $\beta=0.8$.

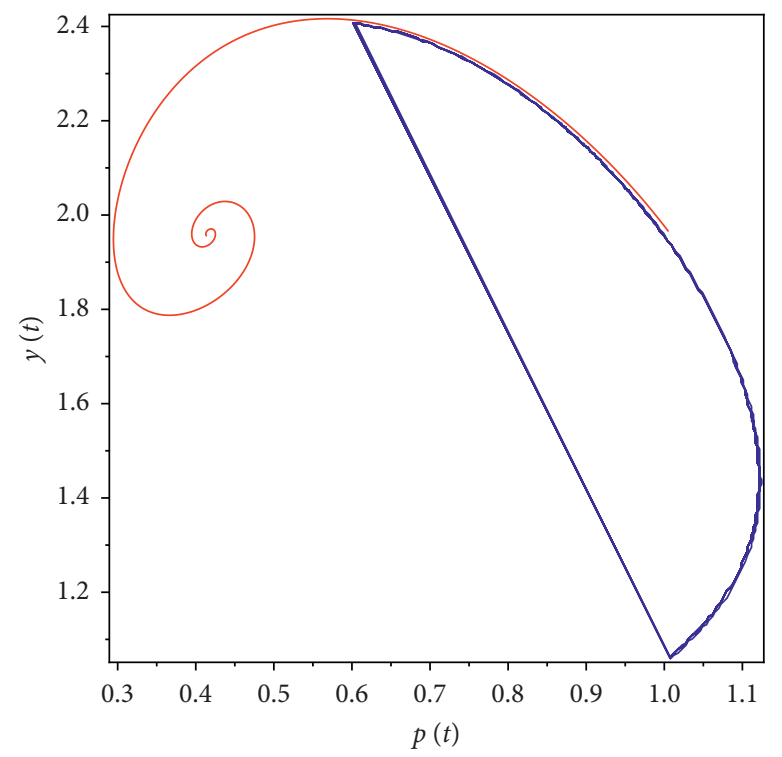

(a)

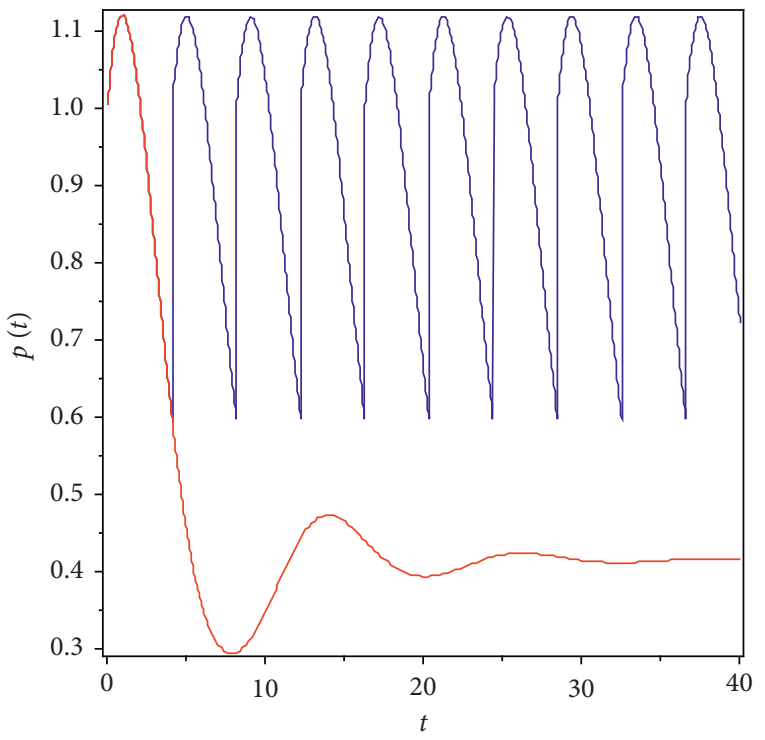

(b)

FIgURE 6: Continued. 


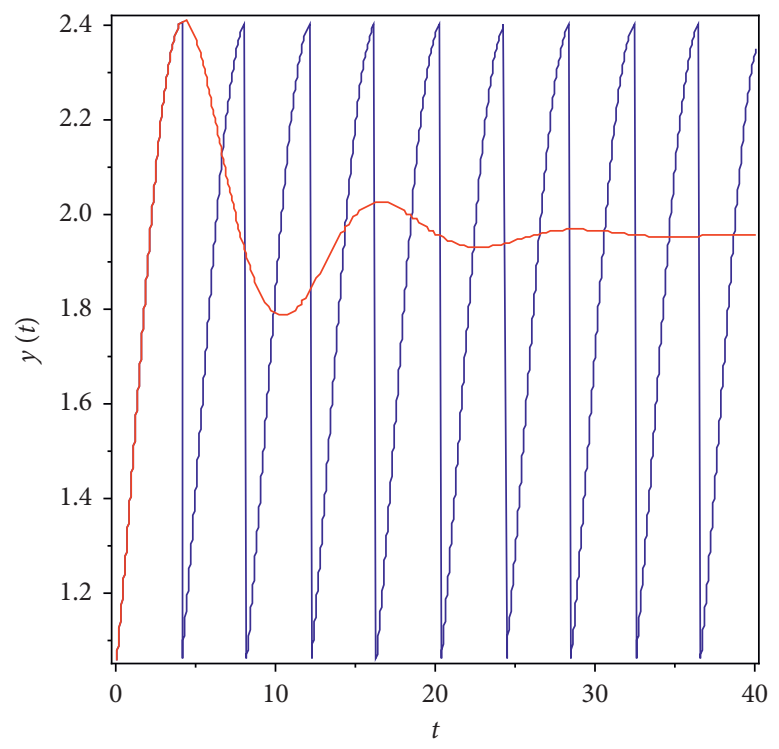

(c)

Figure 6: Phase portrait and time series of system $k=1.8, a=0.15, \tau=0.6, \bar{p}=0.6, \theta=0.8, \delta=0.65$, and $\beta=0.4$.

On the contrary, according to the expression of Poincaré map, we obtain that $F_{M}^{3}(0)>0$. So, $\exists y \in\left(0, y_{m}^{+}\right)$which makes $F_{M}^{3}(y)=y$. This indicates that system (7) has order-3 period solution with $(\bar{p}+(\tau / 1+\theta \bar{p}), y)$ as the initial point.

In the same way, we can prove that if $F_{M}^{k-1}\left(y_{A}\right)<y_{m}^{+}$, $F_{M}\left(y_{m}^{+}\right)=y_{A} Z$, system (7) possesses the order- $k(k \geq 2)$ periodic solution.

\section{Numerical Simulations}

An impulsive semidynamic system of plankton and herbivore is established in this paper. The numerical simulation illustrates the correctness of our arguments.

We set the parameter value as $k=1.8, a=0.15, \tau=0.6$, $\bar{p}=0.6, \theta=0.8, \delta=0.25$, and $\beta=0.8$, and obtain that system (7) possesses a globally asymptotically stable order-1 periodic solution if $F_{M}\left(y_{A}\right)>y_{A}$ (Figure 5(a)). Furthermore, let $k=1.8, a=0.15, \tau=0.6, \quad \bar{p}=0.6, \theta=0.8$, $\delta=0.65$, and $\beta=0.4$. We get that model (7) has unique order-1 periodic solution when $F_{M}\left(y_{A}\right)<y_{A}$, and it is globally asymptotically stable (Figure 6(a)). Figures 5(b) and $5(\mathrm{c})$ are time series diagram when $F_{M}\left(y_{A}\right)>y_{A}$, and Figures $6(\mathrm{~b})$ and $6(\mathrm{c})$ are time series diagram when $F_{M}\left(y_{A}\right)<y_{A}$. In addition, the red trajectories represent the phase portrait and time series of system (7) without pulses. By comparison, we get that plankton and herbivore can survive stably under effective control.

From Figure 7, we get that although the trajectories start from different initial points, they all converge to the same order-1 periodic solution. And the trajectory eventually stabilizes, which implies that the order-1 periodic solution is globally asymptotically stable.

When herbivores are absent and $\delta=0$, we get the expression of the boundary period solution. Through analysis,
(7) when $F_{M}\left(y_{A}\right)<y_{A}$. And the parameters are fixed as

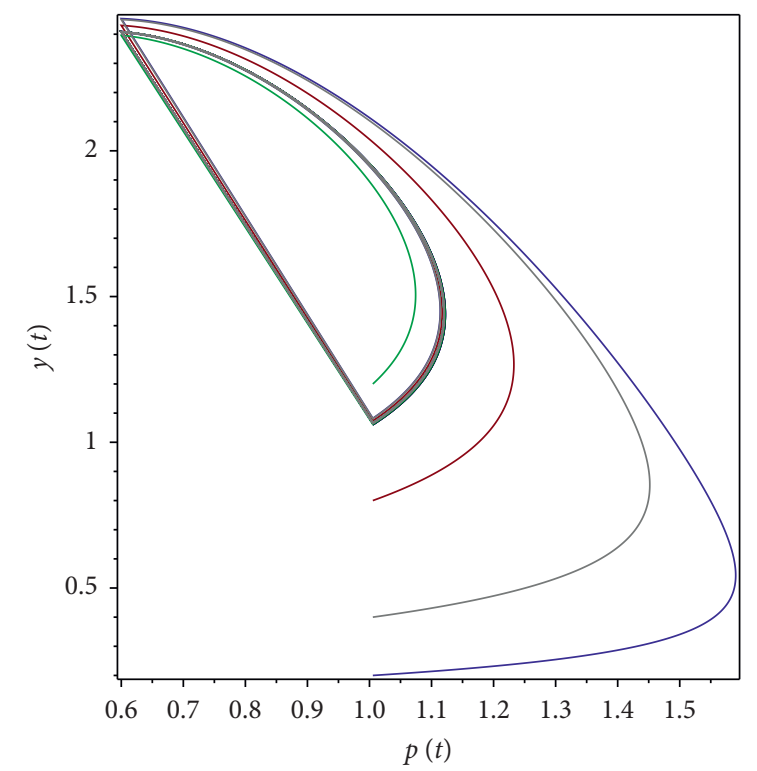

FIgUre 7: Periodic solution of system (7) at different initial points. And the parameters are fixed as $k=1.8, a=0.15, \tau=$ $0.6, \bar{p}=0.6, \theta=0.8, \delta=0.65$, and $\beta=0.4$.

the boundary periodic solution is asymptotically stable under certain conditions. Thus, the rationality of the plankton release method can be obtained. After no longer harvesting herbivores, that is, when $\delta=0$, we get that the number of herbivores increases and eventually approaches a stable value, and the plankton population will oscillate periodically from Figures $8(\mathrm{a})-8(\mathrm{c}))$. Although herbivores will increase temporarily, they will be in equilibrium after a period of time, and the number will no longer increase. However, pulse control allows herbivores to grow periodically, resulting in sustainable harvests and thus greater gains. 


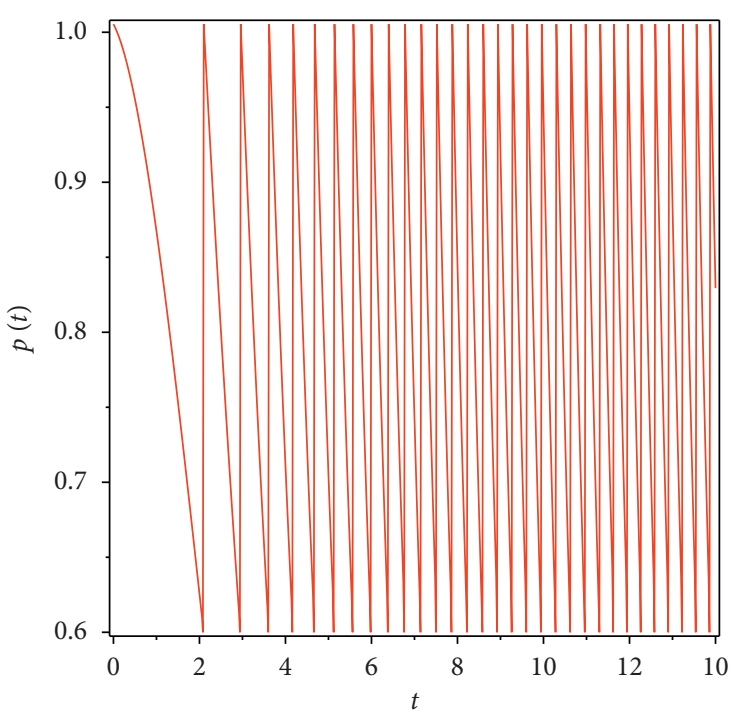

(a)

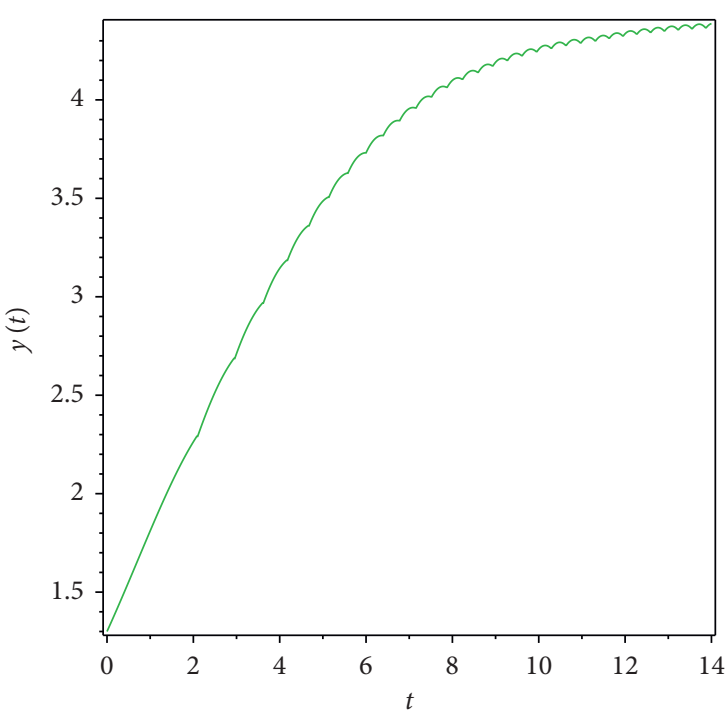

(b)

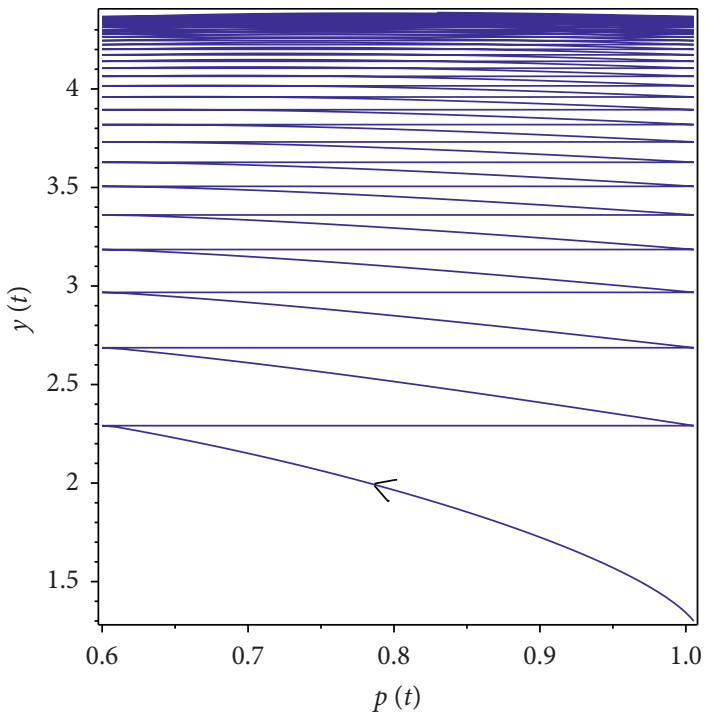

(c)

Figure 8: Phase portrait and time series when not hunting herbivores. And the parameters are fixed as $k=1.8, a=0.15, \tau=0.6, \bar{p}=0.6, \theta=0.8$, and $\delta=0$.

Thus, we get the rationality and necessity of taking pulse control of this paper.

\section{Conclusion}

In this paper, an impulsive semidynamic system for the relationship between plankton and herbivores is proposed. We adopt the method of Poincaré map to comprehensively analyze the dynamic properties of system (7). Moreover, state feedback control is adopted to enable plankton and herbivores to survive stably.

Firstly, the model is introduced, and the conditions for the existence and stability of the equilibrium point of the model are analyzed. Secondly, we define the Poincare map of the model and study its main properties such as monotonicity, continuity, fixed point, and extremum. Then, we discuss the existence and local and global stability of order-1 periodic solution and give sufficient conditions for the local stability of the boundary periodic solution. In addition, the existence of the order- $k(k \geq 2)$ periodic solution is obtained through the proof of the existence of the order-3 periodic solution. Finally, our theoretical results are verified by numerical simulation.

From biological perspective, periodic solutions and state feedback control have important roles in ecology and fisheries. When the plankton is reduced to a certain amount, a state feedback control strategy of increasing plankton and hunting herbivores is adopted. In the end, a periodic solution can be formed, and the plankton and herbivore are stabilized. The analysis of the boundary periodic solution verifies the rationality and necessity of the pulse form used in this paper. In particular, if not controlled, the number of 
plankton will continue to decrease, which will reduce the food for herbivores and affect yield.

We summarize some of the innovations in this paper: (1) the impulse semidynamic system studied in this paper uses the new form of pulse, and the control parameters are changed according to the actual situation. This makes the system have more complex dynamic properties, which is worthy of our in-depth study. (2) In this paper, the Poincaré map is defined and used as a tool to carry out more comprehensive dynamic analysis of the system. For example, by analyzing the impulse function, the precise range of the pulse and phase set is obtained, and a more comprehensive and detailed study of periodic solutions is carried out. Compared with [43], we not only prove the existence and stability of the order-1 periodic solution but also give the necessary and sufficient condition for the global stability of the order-1 periodic solution and the existence conditions of the order- $k$ $(k \geq 2)$ periodic solution. (3) Through the analysis and numerical simulation of the model, the practical biological significance of its complex dynamics is revealed. When $k>2 p^{*}+2$, the system has more complicated dynamic properties. In future research work, we will further discuss the complex dynamics of the system and optimize to obtain maximum benefits.

\section{Data Availability}

The data used to support the findings of this study are available from the corresponding author upon request.

\section{Conflicts of Interest}

The authors declare that there are no conflicts of interest regarding the publication of this paper.

\section{Authors' Contributions}

All authors read and approved the final manuscript.

\section{Acknowledgments}

This work was supported by the National Natural Science Foundation of China (11371230), the SDUST Research Fund (2014TDJH102), the Shandong Provincial Natural Science Foundation, China (ZR2015AQ001), and Joint Innovative Center for Safe and Effective Mining Technology and Equipment of Coal Resources, SDUST Innovation Fund for Graduate Students (SDKDYC170225).

\section{References}

[1] J. T. Lehman, "Release and cycling of nutrients between planktonic algae and herbivores," Limnology and Oceanography, vol. 25, no. 4, pp. 620-632, 1980.

[2] S. Busenberg, S. K. Kumar, P. Austin, and G. Wake, "The dynamics of a model of a plankton-nutrient interaction," Bulletin of Mathematical Biology, vol. 52, no. 5, pp. 677-696, 1990.

[3] Z. Jiang, W. Zhang, J. Zhang, and T. Zhang, "Dynamical analysis of a phytoplankton-Zooplankton system with harvesting term and holling III functional response,"
International Journal of Bifurcation and Chaos, vol. 28, no. 13, Article ID 1850162, 2018.

[4] T. Daufresne and M. Loreau, "Plant-herbivore interactions and ecological stoichiometry: when do herbivores determine plant nutrient limitation," Ecology Letters, vol. 4, no. 3, pp. 196-206, 2010.

[5] Z. Zhong, L. Pang, and X. Song, "Optimal control of phytoplanktoncfish model with the impulsive feedback control," Nonlinear Dynamics, vol. 88, no. 3, pp. 2003-2011, 2017.

[6] A. Sharma, A. K. Sharma, and K. Agnihotri, "Analysis of a toxin producing phytoplanktonczooplankton interaction with holling iv type scheme and time delay," Nonlinear Dynamics, vol. 81 , no. 1-2, pp. 13-25.

[7] S. Tang, J. Liang, Y. Tan, and R. A. Cheke, "Threshold conditions for integrated pest management models with pesticides that have residual effects," Journal of Mathematical Biology, vol. 66, no. 1-2, pp. 1-35, 2013.

[8] Y. Li, H. Cheng, and Y. Wang, "A lycaon pictus impulsive state feedback control model with allee effect and continuous time delay," Advances in Difference Equations, vol. 2018, no. 1, p. 367, 2018.

[9] H. Liu and H. Cheng, "Dynamic analysis of a preycpredator model with state-dependent control strategy and square root response function," Advances in Difference Equations, vol. 2018, no. 1, p. 63, 2018.

[10] D. Li, H. Cheng, and Y. Liu, "Dynamic analysis of Beddington-DeAngelis predator-prey system with nonlinear impulse feedback control," Complexity, vol. 2019, Article ID 5308014, 13 pages, 2019.

[11] X. Liu, Y. Li, and W. Zhang, "Stochastic linear quadratic optimal control with constraint for discrete-time systems," vol. 228, pp. 264-270, 2014.

[12] J. Lou, Y. Lou, and J. Wu, "Threshold virus dynamics with impulsive antiretroviral drug effects," Journal of Mathematical Biology, vol. 65, no. 4, pp. 623-652, 2012.

[13] T. Zhang, X. Meng, and T. Zhang, "Global dynamics of a virus dynamical model with cell-to-cell transmission and cure rate," Computational and Mathematical Methods in Medicine, vol. 2015, no. 45C48, pp. 1-8, 2015.

[14] Z. Chang, X. Meng, and T. Zhang, "A new way of investigating the asymptotic behaviour of a stochastic sis system with multiplicative noise," Applied Mathematics Letters, vol. 87, pp. 80-86, 2019.

[15] W. Lv and F. Wang, "Adaptive tracking control for a class of uncertain nonlinear systems with infinite number of actuator failures using neural networks," Advances in Difference Equations, vol. 2017, no. 1, p. 374, 2017.

[16] Z. Shi, Y. Li, and H. Cheng, "Dynamic analysis of a pest management smith model with impulsive state feedback control and continuous delay," Mathematics, vol. 7, no. 7, p. 591, 2019.

[17] Y. Wang, H. Cheng, and Q. Li, "Dynamic analysis of wild and sterile mosquito release model with poincaré map," Mathematical Biosciences and Engineering, vol. 6, no. 16, pp. 7688-7706, 2019.

[18] Z. Shi, H. Cheng, Y. Liu, and Y. Wang, "Optimization of an integrated feedback control for a pest management predatorprey model," Mathematical Biosciences and Engineering, vol. 16, no. 6, pp. 7963-7981, 2019.

[19] Z. Shi, H. Cheng, Y. Liu, and Y. Li, "A cydia pomonella integrated management predator-prey model with smith growth and linear feedback control," IEEE Access, vol. 7, no. 1, pp. 126066-126076, 2019. 
[20] L. Zhang, Y. Wang, C. M. Khalique, and Y. Bai, "Peakon and cuspon solutions of a generalized Camassa-Holm-Novikov equation," Journal of Applied Analysis and Computations, vol. 8, no. 6, pp. 1938-1958, 2018.

[21] A. d'Onofrio, "On pulse vaccination strategy in the SIR epidemic model with vertical transmission," Applied Mathematics Letters, vol. 18, no. 7, pp. 729-732, 2005.

[22] W. Zhang, X. Meng, and Y. Dong, "Periodic solution and ergodic stationary distribution of stochastic siri epidemic systems with nonlinear perturbations," Journal of Systems Science and Complexity, vol. 32, pp. 1-21, 2019.

[23] W. Zhao, J. Liu, M. Chi, and F. Bian, "Dynamics analysis of stochastic epidemic models with standard incidence," $A d$ vances in Difference Equations, vol. 2019, no. 1, 2019.

[24] X. Meng, F. Li, and S. Gao, "Global analysis and numerical simulations of a novel stochastic eco-epidemiological model with time delay," Applied Mathematics and Computation, vol. 339, pp. 701-726, 2018.

[25] W. Wang, W. Ma, and Z. Feng, "Complex dynamics of a time periodic nonlocal and time-delayed model of reaction-diffusion equations for modeling $\mathrm{CD}^{4+} \mathrm{T}$ cells decline," Journal of Computational and Applied Mathematics, vol. 367, Article ID 112430, 2020.

[26] J. Wang, H. Cheng, H. Liu, and Y. Wang, "Periodic solution and control optimization of a prey-predator model with two types of harvesting," Advances in Difference Equations, vol. 2018, no. 1, p. 41, 2018.

[27] J. Wang, H. Cheng, X. Meng, and B. S. A. Pradeep, "Geometrical analysis and control optimization of a predator-prey model with multi state-dependent impulse," Advances in Difference Equations, vol. 2017, no. 1, p. 252, 2017.

[28] Y. Li, H. Cheng, J. Wang, and Y. Wang, "Dynamic analysis of unilateral diffusion Gompertz model with impulsive control strategy," Advances in Difference Equations, vol. 2018, no. 1, p. 32, 2018.

[29] T. Zhang, X. Meng, Y. Song, and T. Zhang, "A stage-structured predator-prey si model with disease in the prey and impulsive effects," Mathematical Modelling and Analysis, vol. 18, no. 4, pp. 505-528, 2013.

[30] F. Wang, B. Chen, S. Yumei, and C. Lin, "Finite time control of switched stochastic nonlinear systems," Fuzzy Sets and Systems, vol. 365, pp. 140-152, 2019.

[31] Z. Shi, J. Wang, Q. Li, and H. Cheng, "Control optimization and homoclinic bifurcation of a preycpredator model with ratio-dependent," Advances in Difference Equations, vol. 2019, no. 1, p. 2, 2019.

[32] Y. Li, Y. Li, Y. Liu, and H. Cheng, "Stability analysis and control optimization of a prey-predator model with linear feedback control," Discrete Dynamics in Nature and Society, vol. 2018, p. 12, 2018.

[33] L. Zhang and M. Khalique, "Classification and bifurcation of a class of second-order odes and its application to nonlinear pdes," Discrete and Continuous Dynamical Systems-Series S, vol. 11, pp. 777-790, 2017.

[34] Y. Liu, H. Dong, and Y. Zhang, "Solutions of a discrete integrable hierarchy by straightening out of its continuous and discrete constrained flows," Analysis and Mathematical Physics, vol. 9, no. 2, pp. 1-17, 2018.

[35] Z. Zhang and J. Luo, "Multiple periodic solutions of a delayed predatorcprey system with stage structure for the predator," Nonlinear Analysis: Real World Applications, vol. 11, no. 5, pp. 4109-4120, 2010.

[36] Z. Zhang and J. Cao, "Periodic solutions for complex-valued neural networks of neutral type by combining graph theory with coincidence degree theory," Advances in Difference Equations, vol. 2018, no. 1, p. 261, 2018.

[37] A. A. Andronov, E. A. Leontovich, I. I. Gordon, and A. G. Maier, Qualitative Theory of Second Order Dynamic Systems, Physics Today, vol. 27, no. 8, pp. 53-54, 1974.

[38] E. M. Bonotto and M. Federson, "Limit sets and the poincarcbendixson theorem in impulsive semidynamical systems," Journal of Differential Equations, vol. 244, no. 9, pp. 23342349, 2008.

[39] S. Kaul, "On impulsive semidynamical systems," Journal of Mathematical Analysis and Applications, vol. 150, no. 1, pp. 120-128, 1990.

[40] A. S. Matveev and A. V. Savkin, Qualitative Theory of Hybrid Dynamical Systems I, Springer, Basel, Switzerland, 2000.

[41] F. Liu, "Continuity and approximate differentiability of multisublinear fractional maximal functions," Mathematical Inequalities \& Applications, vol. 21, no. 1, pp. 25-40, 2018.

[42] L. Ntr and j.d. Murray, "Mathematical biology. i. an introduction," Photosynthetica, vol. 40, no. 3, p. 414, 2002.

[43] D. Fang, Y. Pei, Y. Lv, and L. Chen, "Periodicity induced by state feedback controls and driven by disparate dynamics of a herbivorecplankton model with cannibalism," Nonlinear Dynamics, vol. 90, no. 5, pp. 1-16, 2017.

[44] Y. Tian, S. Tang, and R. A. Cheke, "Nonlinear state-dependent feedback control of a pest-natural enemy system," Nonlinear Dynamics, vol. 94, no. 3, pp. 2243-2263, 2018. 